\title{
Optimization of parameters effecting the heat recovery from a sand casting process
}

\author{
V S GopalaKrishna ${ }^{1}$, K Prakash Marimuthu ${ }^{1}$ \\ ${ }^{1}$ Department of Mechanical Engineering, Amrita School of Engineering, Bengaluru, Amrita Vishwa Vidyapeetham, India.
}

\begin{tabular}{l} 
Article Info \\
\hline Article history: \\
Received Aug 13, 2018 \\
Revised Aug 29, 2018 \\
Accepted Oct 26, 2018 \\
\hline
\end{tabular}

\section{Keyword:}

Energy

Casting

Particle Swarm optimization conservation

\begin{abstract}
Energy crises has gripped the world to a large extent. Researchers are experimenting on newer and newer avenues to produce energy efficiently, economically. Energy saved is actually twice produced. Energy conservation is the need of the hour. Keeping this in mind the authors of the paper have developed a method to harness the heat that gets wasted during the casting process. Casting is basically one of the manufacturing processes were molten metal is poured into mould and then solidified. The solidifying metal takes the shape of the mould. Huge amount of energy is required for the melting of the raw material. When the metal solidifies it gives way the heat. In the present work the mould is modified such that the raw material that will be used for subsequent process gets pre heated by the heat that emanates due to the solidification process. A number of factors influence the amount of heat that is recovered. Here attempt is made to find out the factors that influence the pre heat of raw material and also using Particle swarm optimization technique the factors are optimized so that the heat recovered is maximised. The implementation of the process is simple and the gains are enormous in terms of the energy that is going to be saved.
\end{abstract}

\section{Corresponding Author:}

Second Author,

Departement of Mechanical Engineering,

Amrita School of Engineering, Bengaluru,

Amrita Vishwa Vidyapeetham, India.

Email: k_prakash@blr.amrita.edu

\section{Introduction}

Development of a nation primarily depends on Energy, India being no exception, is short of fossil fuels and needs to discover more sustainable energy alternatives [1] for effective transport as well as for manufacturing \& industrial needs [2]. The Energy utilization \& consumption depends upon factors such as economy \& government but may reflect into the change in the living standards, globalization etc but their effects on nature \& natural resources mainly imply a need for greater sustainable energy resources such as Solar [3], wind ,wave energy ,tidal energy etc. [4]. These sustainable energy sources must meet the demands of swelling population, rapid technological and industrial developments [5]. We can see that Industrial areas consume lots of energy and much of it is supplied through unsustainable resources. Unsustainable supplies are Thermal plant, Nuke plant etc. Most of the industries are energy intensive industries. Energy intensive industries play a central role in a country's development [6]. Foundry is one such energy intensive industry wherein energy is wasted a lot. Foundry has different stages involved. But only in melting stage it is estimated that $55 \%$ of the total energy is consumed. 


\section{ENERGY PROFILE OF EACH PROCESS OF CASTING}

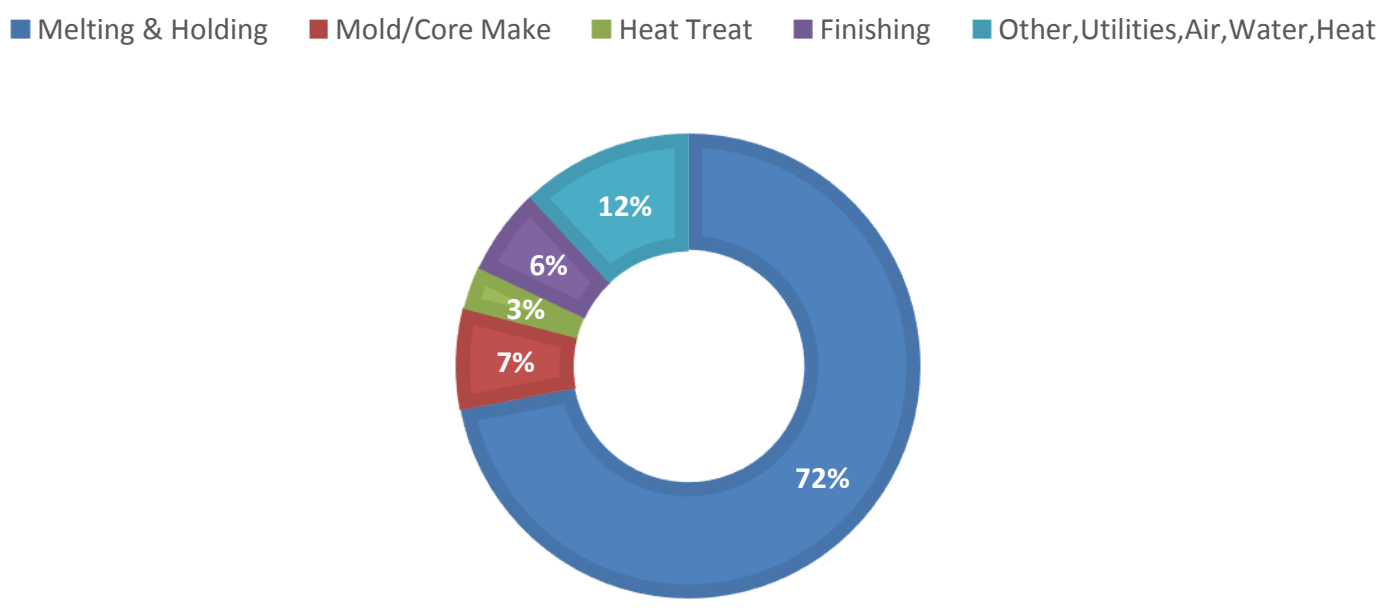

Figure 1 showing the energy profile of each process in casting [7]

Although this high consumption has been alarming for metal casters, break through efforts on reducing the energy consumption and waste energy recovery are in progress. The low thermal efficiencies in the energy sustenance is contributed by inefficient furnaces currently used in the industry. But currently emerging technologies such as solar melting, microwave melting, infrared heating, plasma heating offer huge energies to melt the metal effectively accompanying sustainable methods, although such methods require huge capital and space for installation with increased maintenance costs [8][9].Therefore for such new technological adaptations there must some support from government which would help the industries use the sustainable methods and help the environment and as well as curb the waste produced from non-renewable sources and save the long term capital.

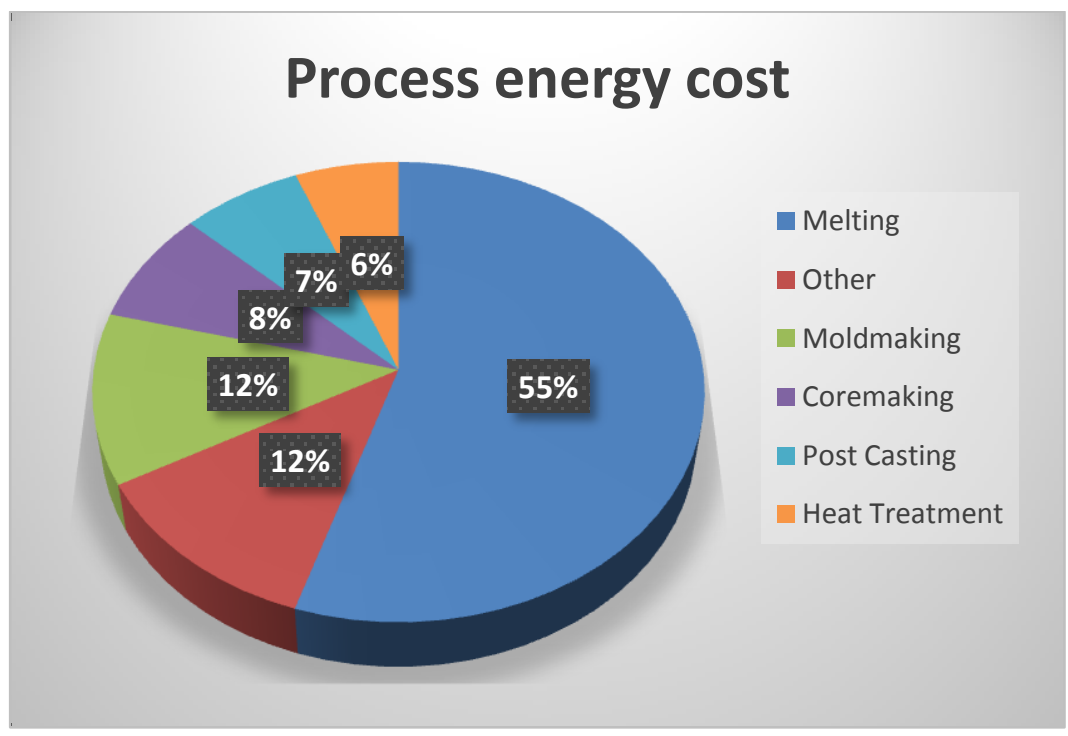

Figure 2 Showing the Process energy costs in casting [9]

Foundry being one of the most energy intensive process still continues to use low energy efficient processes [10]. In the present work mathematical modelling was done for a casting process from which the heat is recovered to use it for subsequent processes. The details of the work are given in the following sections. 


\section{Mathematical Modelling}

Mathematical modelling is a method of converting the real life problem into mathematical expressions which under constraints gives the results just like a real model, which helps us to analyse the problem and develop a better solution [11]. These mathematical models need to be optimized for better outputs from a range of inputs. Mathematical modelling is an important step taken in application oriented mathematical training which helps in handling the challenges of real life problems [12], [13] [14]. Mathematical modelling is used in various fields such as Anthropology, Archaeology, Architecture, Artificial Intelligence, Arts, Astronomy, Biology, Chemical Engineering, Chemistry, Computer science, Criminalistic Science, Electrical Engineering, Economics, Finance, Fluid-mechanics, Geoscience, Linguistics, Optimal-routing, Material sciences, Mechanical engineering, Medicine, metrology, Music, Meteorology quite more [15].

\section{Optimization}

Optimization of a problem is extracting the best possible solution from the given range of inputs. This can be highest or lowest possible for a given problem. Optimization methods generally involve in generating and selecting a best solution from a set of available options [16], by systematically choosing input values from a domain and computing the output of the function and recording the best output values found during the process [17]. Some optimization methods being Genetic algorithm, Memetic algorithm, Dynamic relaxations, Gravitational search algorithm, Reactive Search Algorithm (RSO) etc.

\section{Experiment}

In the present research optimizing the objective function obtained from the experiments conducted [4] is done using PSO. The experiment briefly included considering the sand-casting method and to harness the waste energy. The setup is shown in Figure 3. The waste energy is released in the form of heat to the sand in contact with molten metal[14]. The setup involves flasks (cope and drag), thermocouples, insulators, raw material (used for preheating) and cavity in which molten metal is poured. Now when the molten metal is poured into the cavity the waste heat released while the metal is solidifying is released to the surroundings and that heat is used as another source to heat the raw material (simply preheating the raw material). The insulator surrounding is used to prevent the heat from escaping from the flask. The thermocouples are used to obtain the temperatures from the regions where the temperature is needed to be known. The temperatures are recorded at $10 \mathrm{~mm}$ from the cavity, in the middle of the raw material (scraps) and after the insulation's outer wall.

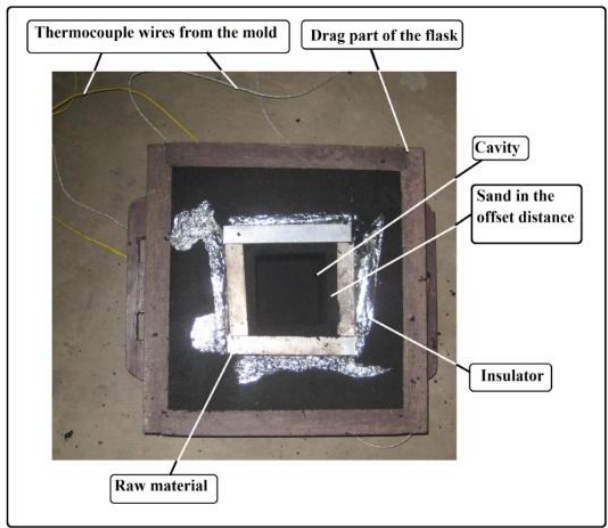

Figure 3 Experimental setup-showing raw material, insulator, cavity for charge, Thermocouple wires for measuring temperature, drag part [7]

Now the experimental findings were noted and the results were tabulated. Upon analysis it is found that the parameters on which the temperature distribution depends are offset distance, moisture content of the sand, insulator thickness. By regression analysis the relation between the three parameters and the output temperature was found. This objective function is optimized to find the value at which maximum temperature is obtained.

The experiment demonstrates a process in which the escaping heat is used to pre-heat raw material (scraps and billets), this preheated material is going to act as a charge for the furnace, this preheat will reduce energy 
consumption in the furnace. The aim of our project is to optimize the mathematical model of the above explained setup \& find out the optimal Preheat temperature.

The methodology involved taking the charge material and embedding it into the mould in such a way that all the 3 above stated parameters are constant, the raw materials are surrounded by insulators to prevent the loss of heat from the raw materials ,the thermocouples are provided to measure temperature at appropriate positions[7]. While conducting the experiment various factors found to be influencing the heat recovered, moisture content of the sand by weight $\left(\mathrm{m}_{\mathrm{s}}\right)$, offset distance $\left(\mathrm{d}_{\mathrm{o}}\right)$, which is the distance of the raw material from the cavity wall, and the thickness of the insulator $\left(\mathrm{t}_{\mathrm{i}}\right)$ that surrounds the raw material to curb the heat loss and various factors such as size of the casting, temperature of the pouring molten metal were not considered by the experimenter because of the reasons stated :

- In case of the size of the casting, the amount of raw material put around the cavity changes proportionately while changing size of casting, leading to a balanced absorption of heat per unit mass.

- In case of molten metal pouring temperature, for the given alloy/metal, it is fixed depending on the metallurgical conditions.

Hence only 3 factors were considered, the design factors and respective levels are given in Table 1.

Table 1 giving the design factors \& respective levels [7]

\begin{tabular}{|c|c|l|l|}
\hline \multirow{2}{*}{ Levels } & \multicolumn{3}{|c|}{ Factors } \\
\cline { 2 - 4 } & $\begin{array}{l}\text { Moisture content of } \\
\text { sand }(\% \text { by weight }) \\
\left(\mathrm{m}_{\mathrm{s}}\right)\end{array}$ & $\begin{array}{l}\text { Offset } \\
\left(\mathrm{d}_{\mathrm{o}}\right)(\mathrm{mm})\end{array}$ & $\begin{array}{l}\text { Insulator } \\
\text { thickness }\left(\mathrm{t}_{\mathrm{j}}\right)(\mathrm{cm})\end{array}$ \\
\hline 1 & 3.5 & 10 & 4 \\
\hline 2 & 5 & 20 & 10 \\
\hline 3 & 6.5 & 30 & 16 \\
\hline
\end{tabular}

Using design of experiments the number of experiments to capture the effect of input variables on the output variables can be reduced [18]. Using Minitab 16.0, a set of 16 experiments were by using Box-Behnken method. The heat recovered from the cooling molten metal was measured. The experimental design and their respective responses are shown in Table2

Table 2 experimental design responses [7]

\begin{tabular}{|l|l|l|l|l|}
\hline Experiment no. & $\begin{array}{l}\text { Factors (as per } \\
\text { designed } \\
\text { experiments) }\end{array}$ & & & $\begin{array}{l}\text { Response (by } \\
\text { experimenting) preheat } \\
\text { from } \\
\text { temperature }\end{array}$ \\
\hline & $\mathrm{m}_{\mathrm{s}}(\%)$ & $\mathrm{t}_{\mathrm{i}}(\mathrm{cm})$ & $\mathrm{d}_{\mathrm{o}}(\mathrm{mm})$ & $\begin{array}{l}(\mathrm{T}), \quad \text { (degree } \\
\text { Celsius) }\end{array}$ \\
\hline 1 & 3.5 & 4 & 20 & 104 \\
\hline 2 & 5 & 16 & 10 & 172 \\
\hline 3 & 5 & 10 & 20 & 106 \\
\hline 4 & 6.5 & 10 & 30 & 94 \\
\hline 5 & 3.5 & 16 & 20 & 114 \\
\hline 6 & 6.5 & 16 & 20 & 105 \\
\hline 7 & 5 & 10 & 20 & 106 \\
\hline 8 & 5 & 10 & 20 & 108 \\
\hline 9 & 6.5 & 10 & 10 & 147 \\
\hline 10 & 6.5 & 4 & 20 & 97 \\
\hline 11 & 5 & 4 & 30 & 93 \\
\hline 12 & 3.5 & 10 & 10 & 156 \\
\hline 13 & 5 & 16 & 30 & 96 \\
\hline 14 & 3.5 & 10 & 30 & 96 \\
\hline
\end{tabular}




\begin{tabular}{|l|l|l|l|l|}
\hline 15 & 5 & 4 & 10 & 143 \\
\hline
\end{tabular}

\section{Regression analysis}

Regression analysis is a statistical process used to estimate the relationship among different factors (one or more inputs) influencing the process and output variable (one response). As per the standard procedure a confidence level of $95 \%$ is chosen or (5\% significant level) with preheat variable as Response (output) and predictor variables as the combination of three main input variables. Combination includes corresponding linear terms, their square (quadratic), two factor interaction term (combined effect of two input variables on the output(T)).

Terms which were found not to be significant at 5\% level were omitted and repeated regression analysis was done at $99.1 \%$ confidence level. This was done to omit any other inconsistencies and for accurate results. Using the regression analysis, the mathematical model was formulated which is given in Eqn. 1[7]

$$
\begin{aligned}
& \text { Preheat Temp }\left({ }^{\circ} \mathrm{C}\right)=220.274-2.25 \times m s+3.20833 \times t i-9.46845 \times d o-.108333 \times t i \times d o+ \\
& .189107 \times d o \times d o
\end{aligned}
$$

This equation is the objective function for the optimization that is being carried out.

This mathematical model is capable of predicting the preheat temperature with an error of $+/-5 \%$.

\section{Particle Swarm Optimization}

There are various optimization techniques employed as listed, but it is difficult to solve the nonlinear equations because it is a tough to make the initial guess. If the convergence of the solution doesn't occur then the algorithm would fail to yield a solution[19]. Hence the convergence \& performance characteristics can be sensitive to the initial guess but PSO, Particle swarm optimization helps in overcoming this problem. Further the problem which we are dealing with is single objective Particle Optimization involving one parameter to be optimized i.e. Temperature function, this method is opposite to that of Multi objective Optimization i.e. involving multiple parameters to be optimized[20] .

Particle Swarm Optimization is a population based stochastic optimization technique inspired by social behaviours such as bird flocking, fish schooling and swarming theory in particular. It is also metaheuristics since it employs the particles to learn themselves, generally these particles being virtual and they cannot think for themselves but enabling metaheuristics it makes them to think as a team by some set of data input and finally they arrive at a solution optimized[21].There are many natural metaheuristic algorithms in addition to earlier mentioned techniques such as Ant Lion Optimization, Dragonfly Algorithm, Grey Wolf Optimization, Sine Cosine Algorithm, and Whale Optimization Algorithm etc which provide optimized solution to a good extent. PSO is one such technique which employs metaheuristics \& stochastic optimization technique.

Stochastic process means a random probability distribution or pattern that may be analysed statistically but may not have exact solutions or may not have precise predictions. Here a simple example is presented on the theory of PSO.

Suppose two people on the opposite banks of the pond would like to know the deepest point in a $2 \mathrm{~d}$ pond, surface of the pond being the datum line, the only way to know the measurements done by individuals is through communication i.e. after each measure the depths they communicate and if first person had measured a depth greater than $2^{\text {nd }}$ person the $2^{\text {nd }}$ person would approach near to the $1^{\text {st }}$ person through some standard distance(assume 1 unit distance).Similarly if the $2^{\text {nd }}$ person measures a greater depth than $1^{\text {st }}$ person, $1^{\text {st }}$ person would approach the $2^{\text {nd }}$ person by unit distance. Finally, by repeated communications between the two the deepest point is found.

This example stated above is a search mechanism, PSO is similar to this. As seen PSO is a very simple concept and it takes few lines of code on the computer and it requires very basic mathematical concepts and its computationally inexpensive in terms of memory and processing capabilities. It has very wide range of application, very effective in solving many real-life problems. Example it has ties with A life (Artificial Life), evolutionary search examples etc. all of which constitute to the ordinary life processes of nature which appear complex to humans. 
PSO uses swarm intelligence, opposed to thinking capabilities of a single being, a collective effort from the members of a swarm gives greater results. Even though the logical thinking of a being can be counted to near zero, the response that a swarm shows in-comparison is great!

In PSO hypothetical or virtual particles are created, with each particle having some characteristic value. Each particle has position, velocity, personal temperature, best temperature, best position. The swarm of particles have Global best as their best value from the group. Now decoding each term, we see that each particle is given positions (range of variables such as moisture content, offset distance etc.) these range of variables are distributed with equal probability among the particles depending upon the number of particles.

Suppose each particle has a definite set of values assigned, now each particle is given some velocity. Velocity, position is governed by some set of simple equations. The working will be dealt later. Now coming to particle, each particle position has some definite temperature-objective-function value. Now corresponding to that the whole group has set of temp_function value, now the optimal value (here it's the maximum temperature of the given set) of that will be the best value of the group and its positional value will be recorded. After this each particle position is given a displacement in a unit time interval updating its position. For this displaced position function value is found and it's compared the other particles to find the Global best function value.

This process repeats till all the particles temperature function value converges to only one value. That value will be the optimal value for the given function. Now let us see the equations and working

$$
v_{i}(t+1)=\frac{x_{i}(t+1)-x_{i}(t)}{t+1-t} \quad E q(2)
$$

simplifying this we get:

This is the position equation of the particle $\mathrm{i}$ at the time $(\mathrm{t}+1)$ :

$$
x_{i}(t+1)=x_{i}+v_{i}(t+1) \quad E q(3)
$$

The velocity equation corresponding to that of the particle $i$ is:

$$
v_{i}(t+1)=w \times v_{i}+r_{1} \times c_{1} \times\left(p_{i}(t)-x_{i}(t)\right)+r_{2} \times c_{2} \times\left(g(t)-x_{i}(t)\right) \quad E q(4)
$$

The first term of the equation is called inertia term, terms starting with $\mathrm{r} 1$, $\mathrm{c} 1$ is called cognitive component, the term starting with $\mathrm{r} 2$, c2 is called social component.c1, $\mathrm{c} 2$ are called acceleration coefficients. $\mathrm{r} 1, \mathrm{r} 2$ are uniformly distributed over $(0,1)[22]$.

Constriction coefficients:

$$
\begin{aligned}
& X=\frac{2 k}{\mid 2-\emptyset-\sqrt{\left(\phi^{2}-4 \varnothing\right) \mid}} \quad E q(5) \\
& \text { where } k=1, \emptyset=\emptyset_{1}+\emptyset_{2} \geq 4, \emptyset_{1}=\emptyset_{2}=2.05 \\
& \text { and } w=X ; c_{1}=X \times \emptyset_{1} ; c_{2}=X \times \emptyset_{2}
\end{aligned}
$$

These equations are utilized in the code format and are fed into to MATLAB, there by curves are plot so to show when the optimal value is reached. Following we see the algorithm for the code execution and its results.

In our problem since our aim is to maximise (optimize) the (output). Preheat temperature, we choose to implement the PSO as a written code in the MATLAB software.

The following is the algorithm for the process:

1) Initialize the required variables.

2) Generate $n$ (say 100) particles and for each of those do the following:

- Assign a random Position drawing uniformly from [3.5 0.0010 .04 ] to [6.5 0.0030 .16 where each coordinate indicates moisture content by \% weight (ms), offset distance(do), thickness(ti) resp. Where the range of values taken by do is .001 to .003 meter. Similarly, others can be understood. 
- Initialize the velocity of each particle to ZERO.

- Evaluate the Temperature (objective function eq. (1)) and assign that as personal best.

- Update the Global Best Temp if the personal best is greater than the global best seen so far.

3) Repeat the following steps 100 times

a. Go through each particle and do the following:
i. Assign a random Velocity
ii. Update the Position based on the velocity given.
iii. Evaluate the Temperature
iv. Update the Personal best
v. Update the global best.

The following illustrates the pseudo code of the PSO algorithm [23]

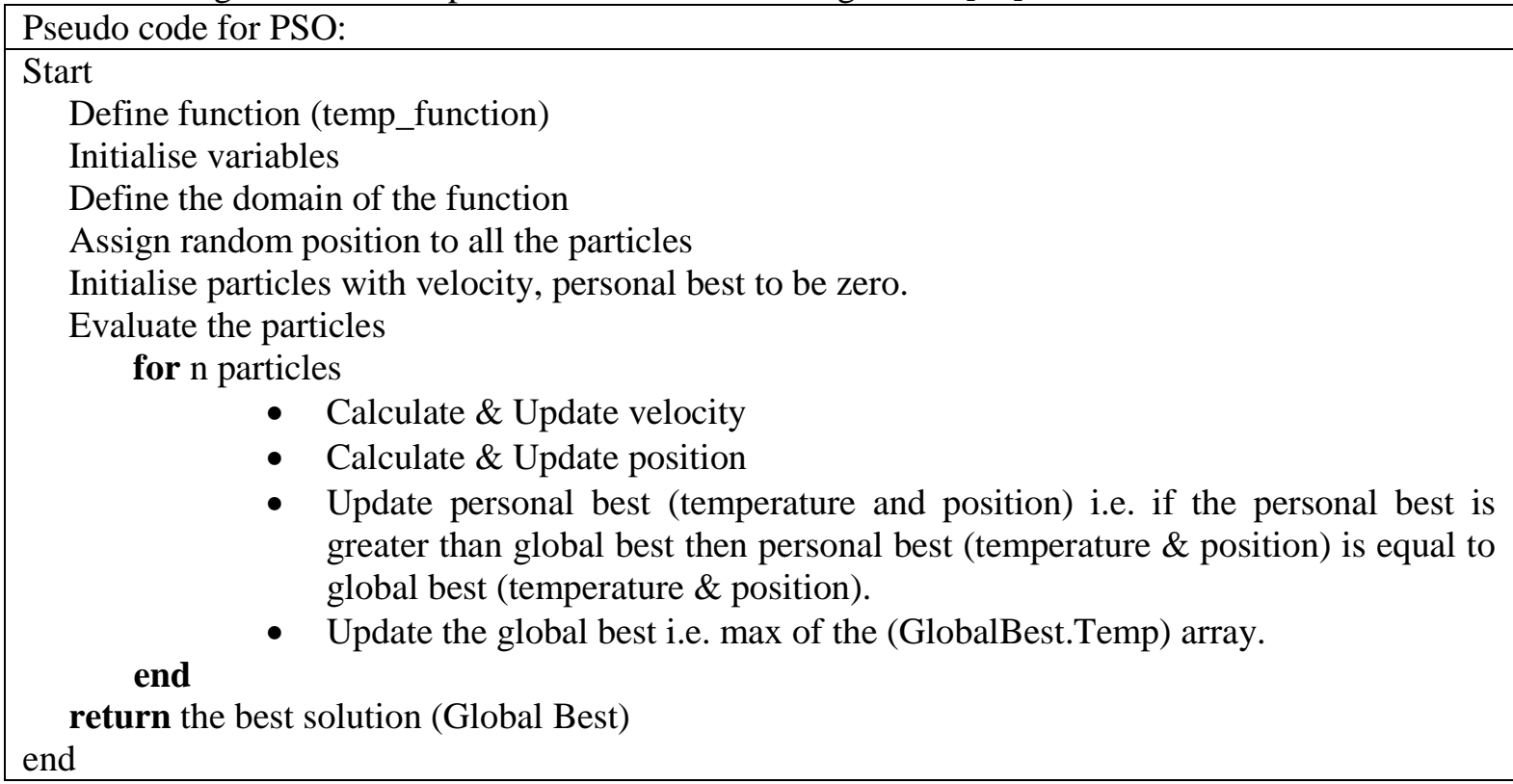

The global best obtained is the response for our query. Plot of Objective function with iterations is

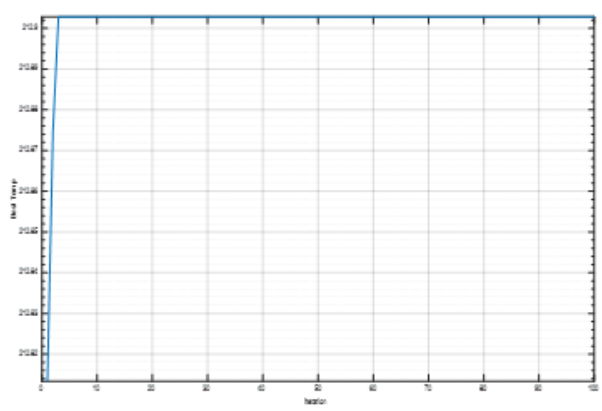

Figure 4 for a swarm size of 10 and iterations being 100 .

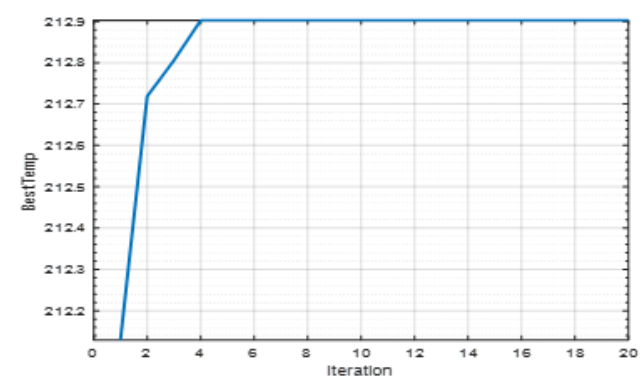

Figure 5: for a swarm size of 10 and iterations being 10 . 


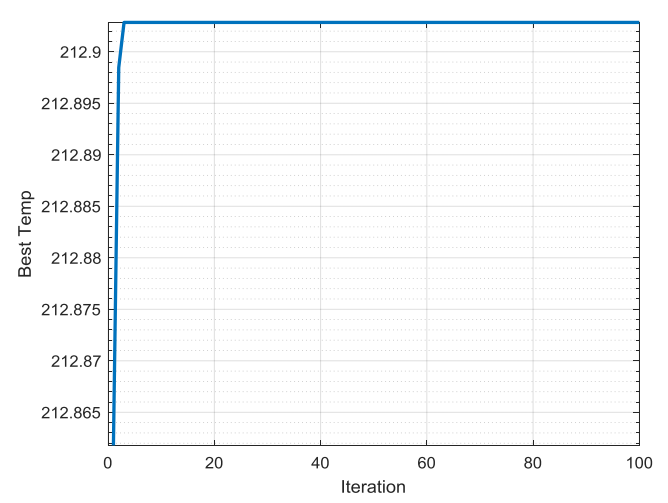

Figure 4 for a swarm size of 100 and iterations being 100.

Therefore, the best parameters for which the temperature is maximized is (number of iterations being 100)

- Moisture content by weight is $3.5 \%$

- Distance offset is $10^{\wedge}--3 \mathrm{~mm}$

- $\quad$ Thickness of the insulator being $.16 \mathrm{~m}$

- $\quad$ The optimized solution being $212.9028^{\circ} \mathrm{C}$

\section{Conclusion}

A novel method of energy harvesting from the solidification process of the casting process has been implemented and the heat recovery capability of the proposed method is established. It is understood that a large amount of energy can be recovered from the process and energy can be saved. The factors that influence the energy recovery was studied and identified. A mathematical model capable of predicting the pre heat temperature that can be gained by the raw material has been formulated using regression analysis. Using Particle swarm optimization technique, the optimized value of the parameters that affect the output was found out .

\section{References}

[1] S. C. Bhattacharya and C. Jana, "Renewable energy in India: Historical developments and prospects," Energy, vol. 34, no. 8, pp. 981-991, Aug. 2009.

[2] J. Pucher, N. Korattyswaropam, N. Mittal, and N. Ittyerah, "Urban transport crisis in India," Transp. Policy, vol. 12, no. 3, pp. 185-198, May 2005.

[3] M. Mwanza, M. KAOMA, C. K. BOWA, N. S. ÇETIN, and K. ÜLGEN, "The Potential of Solar Energy for Sustainable Water Resource Development and Averting National Social Burden in Rural Areas of Zambia," Period. Eng. Nat. Sci., vol. 5, no. 1, pp. 1-7, 2017.

[4] G. Ömeroğlu, "A scrutiny study on wave energy potential and policy in TURKEY," Period. Eng. Nat. Sci., vol. 5, no. 3, pp. 286-297, Oct. 2017.

[5] T. V Ramachandra and G. Hegde, "Energy Trajectory in India: Challenges and Opportunities for Innovation," J. Resour. Energy Dev., vol. 12, no. 1-2, pp. 1-24, Dec. 2015.

[6] J. Zheng and J. Li, "Study on the ecologic network system of energy-intensive industries," Energy Procedia, vol. 5, pp. 1987-1992, 2011.

[7] J. Selvaraja, P. Marimuthu, S. Devanathan, and K. I. Ramachandran, "Mathematical modelling of raw material preheating by energy recycling method in metal casting process," Pollut. Res., vol. 36, no. 3, pp. 598-609, 2017.

[8] D. Zhao, Z. Zhang, X. Tang, L. Liu, and X. Wang, "Preparation of slag wool by integrated waste-heat recovery and resource recycling of molten blast furnace slags: From fundamental to industrial application," Energies, vol. 7, no. 5, pp. 3121-3135, 2014. 
[9] J. Wallace and D. Schwam, "Advanced Melting Technologies: Energy Saving Concepts and Opportunities for the Metal Casting Industry," no. November, pp. 1-42, 2005.

[10] P. Solding and P. Thollander, "Increased Energy Efficiency in a Swedish Iron Foundry Through Use of Discrete Event Simulation," in Proceedings of the 2006 Winter Simulation Conference, 2006, pp. 1971-1976.

[11] A. Neumaier, Modeling Languages in Mathematical Optimization, vol. 88. Boston, MA: Springer US, 2004.

[12] B. M. Turai, C. Satish, and P. M. K, "Mathematical modelling and numerical simulation of forces in milling process Mathematical Modelling and Numerical Simulation of Forces In Milling Process," vol. 20068, 2018.

[13] K. P. Marimuthu, C. S. C. Kumar, and H. P. T. Prasada, "Mathematical modelling to predict the residual stresses induced in milling process," Int. J. Mech. Prod. Eng. Res. Dev., vol. 8, no. 1, pp. $423-$ 428, 2018.

[14] G. Bisio, "Energy recovery from molten slag and exploitation of the recovered energy," Energy, vol. 22, no. 5, pp. 501-509, May 1997.

[15] M. Hodzic, S. Selman, and M. Hadzikadic, "Complex Ecological System Modeling," Period. Eng. Nat. Sci., vol. 4, no. 1, pp. 44-50, Jun. 2016.

[16] A. Çoban, "Optimization of Wear Parameters of Polyamide-6 Composite Materials Filled with Wollastonite Particles," Period. Eng. Nat. Sci., vol. 2, no. 1, pp. 2-7, 2014.

[17] K. Tyagi and K. Tyagi, "A Comparative Analysis of Optimization Techniques," Int. J. Comput. Appl., vol. 131, no. 10, pp. 6-12, Dec. 2015.

[18] B. Durakovic, "Design of experiments application, concepts, examples: State of the art," Period. Eng. Nat. Sci., vol. 5, no. 3, pp. 421-439, 2017.

[19] M. J. Rempe, J. Grønli, T. Thue, J. Mrdalj, A. Marti, J. P. Wisor, J. Mrdalj, A. Marti, P. Meerlo, and J. P. Wisor, "Mathematical Modeling of Sleep State Dynamics in a Rodent Model of Shift Work," Neurobiol. Sleep Circadian Rhythm., 2018.

[20] Z. YILMAZ, M. OKSAR, and F. BASCIFTCI, "Multi-Objective Artificial Bee Colony Algorithm to Estimate Transformer Equivalent Circuit Parameters," Period. Eng. Nat. Sci., vol. 5, no. 3, pp. 271277, Oct. 2017.

[21] U. Can and B. Alatas, "Performance comparisons of current metaheuristic algorithms on unconstrained optimization problems," Period. Eng. Nat. Sci., vol. 5, no. 3, pp. 328-340, 2017.

[22] M. Clerc and J. Kennedy, "The particle swarm - explosion, stability, and convergence in a multidimensional complex space," IEEE Trans. Evol. Comput., vol. 6, no. 1, pp. 58-73, 2002.

[23] S. Ekiz, "Solving Constrained Optimization Problems with Sine-Cosine Algorithm," Period. Eng. Nat. Sci., vol. 5, no. 3, pp. 378-386, 2017. 